4 Gentile DA, Skoner DP. New asthma drugs: small molecule inhaled corticosteroids. Curr Opin Pharmacol 2010; 10: $260-265$

5 Roche N, Postma DS, Colice G, et al. Differential effects of inhaled corticosteroids in smokers/ex-smokers and nonsmokers with asthma. Am J Respir Crit Care Med 2015; 191: 960-964.

6 Global Initiative for Asthma. Global Strategy for Asthma Management and Prevention: updated 2015. Geneva Global Initiative for Asthma, 2015. Available from: http://www.ginasthma.org/local/uploads/files/GINA_Report_ 2015_Aug11.pdf

7 Papi A, Paggiaro PL, Nicolini G, et al. Beclomethasone/formoterol versus budesonide/formoterol combination therapy in asthma. Eur Respir J 2007; 29: 682-689.

8 Papi A, Paggiaro P, Nicolini G, et al. Beclomethasone/formoterol vs fluticasone/salmeterol inhaled combination in moderate to severe asthma. Allergy 2007; 62: 1182-1188.

9 Busse WW, Brazinsky S, Jacobson K, et al. Efficacy response of inhaled beclomethasone dipropionate in asthma is proportional to dose and is improved by formulation with a new propellant. J Allergy Clin Immunol 1999; 104: 1215-1222.

10 Huchon G, Magnussen H, Chuchalin A, et al. Lung function and asthma control with beclomethasone and formoterol in a single inhaler. Resp Med 2009; 103: 41-49.

11 Battaglia S, den Hertog H, Timmers M, et al. Small airways function and molecular markers in exhaled air in mild asthma. Thorax 2005; 60: 639-644.

12 van Veen IH, Sterk PJ, Schot R, et al. Alveolar nitric oxide versus measures of peripheral airway dysfunction in severe asthma. Eur Respir J 2006; 27: 951-956.

13 Clearie KL. McKinlay L, Williamson PA, et al. Fluticasone/salmeterol combination confers benefits in people with asthma who smoke. Chest 2012; 141: 330-338.

14 Thomson NC, Chaudhuri R, Spears M, et al. Poor symptom control is associated with reduced CT scan segmental airway lumen area in smokers with asthma. Chest 2015; 147: 735-744.

15 Brusselle G, Peché R, Van den Brande P, et al. Real-life effectiveness of extrafine beclometasone dipropionate/ formoterol in adults with persistent asthma according to smoking status. Resp Med 2012; 106: 811-819.

\title{
Decreased breath excretion of redox active iron in COPD: a protective failure?
}

To the Editor:

Recent observational and genetic studies have highlighted a potential role for disrupted iron homeostasis in stable and exacerbating chronic obstructive pulmonary disease (COPD). Thus iron deficiency that is not limited to anaemia [1] and single nucleotide polymorphism in the gene encoding iron regulatory protein-2 [2], a key regulatory factor involved in cellular iron turnover and control, have been identified in this population. Disrupted systemic iron homeostasis is likely to limit iron availability for metabolic purposes due to overriding effects on tissue storage rather than on mobilisation and limitation of uptake from the gut. A specific pro-oxidant pool of iron (free or loosely bound ions, which are redox active/catalytic for damaging oxidant production) is measurable in exhaled breath condensate (EBC) [3]. While iron is essential for life, particularly for aerobes, poor or altered iron handling results in adverse effects related to oxidant production, microbial virulence, altered redox signalling events and altered cellular fate, including remodelling. This study was therefore undertaken to gain insight into iron handling in lungs and airways, and the extent to which these processes may be altered in COPD. Studies were undertaken to measure this specific iron pool by the bleomycin method [4] utilising EBC samples collected as previously described [3] and obtained from normal healthy individuals, healthy smokers and patients with COPD (current and ex-smokers). Serum levels of hepcidin and interleukin (IL)-6, known regulators of iron homeostasis, were also measured.

Comparisons of redox active iron content in EBC from age-matched healthy controls, healthy smokers and subjects with COPD, grouped as current and ex-smokers, revealed significant differences between groups (figure 1a). Healthy smokers returned greatly elevated levels of redox active iron in EBC as expected, given that cigarette smoke is known to contain iron and other metallic components [5]. However, no such response was evident in COPD EBC regardless of a current or past smoking history, with values in both groups of the same order as found in samples from healthy controls. There was no significant effect of COPD severity on exhaled redox active iron although a trend was evident (data not shown). In a previous study, which examined redox active iron content in EBC in a variety of subjects (asthma, cardiac surgery and smokers) and settings, the results obtained gave reason to speculate that the presence of this specific and reactive iron pool in EBC may represent an excretory function that may become compromised by 

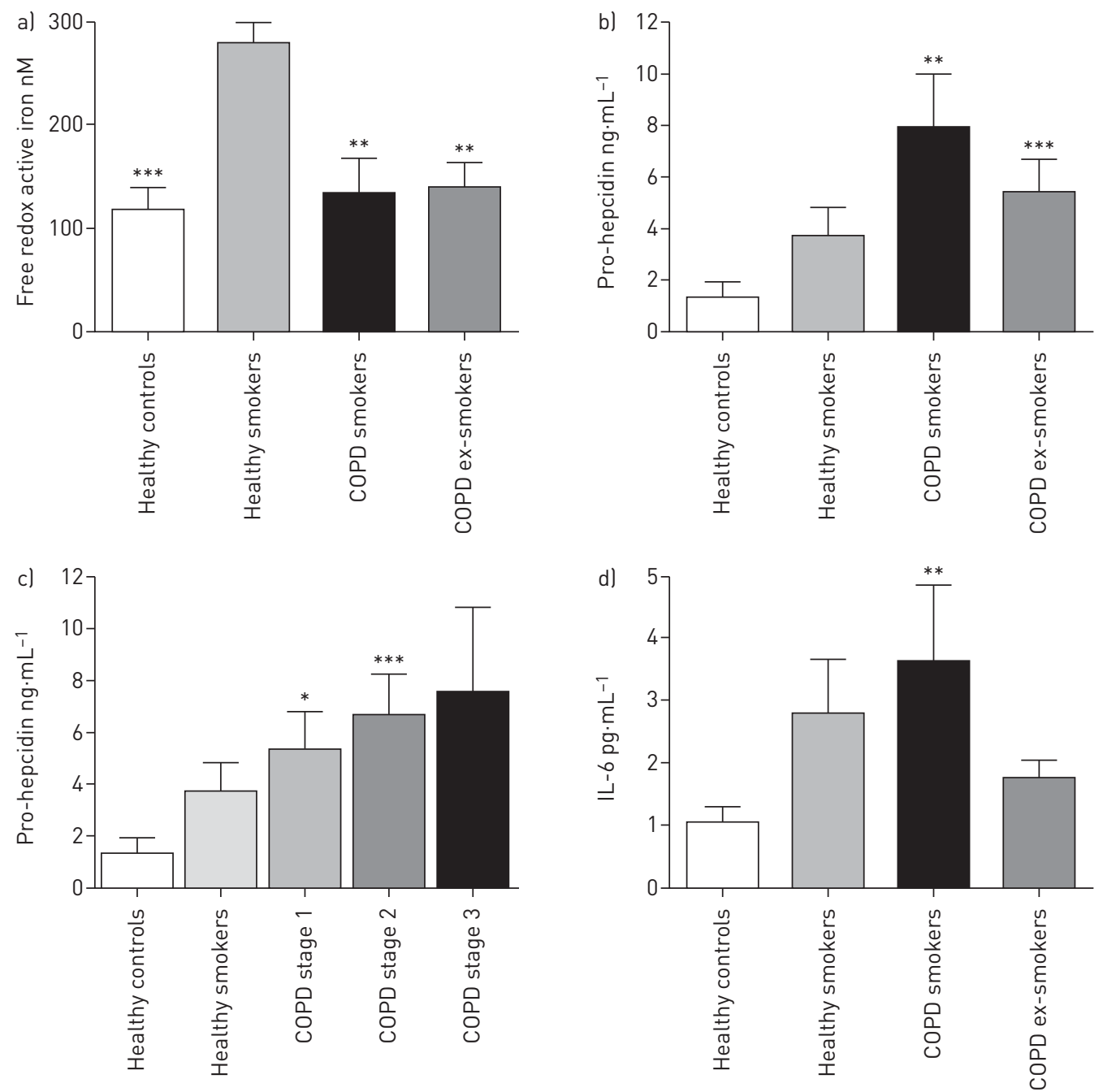

FIGURE 1 a) Redox active iron content in exhaled breath condensate (EBC) from healthy controls $(n=15)$, healthy smokers ( $n=17)$ and subjects with chronic obstructive pulmonary disease (COPD) (current smokers, $n=12$; ex-smokers, $n=18)$. ${ }^{* *}: p<0.001$; and ${ }^{* *}: p<0.01$ when compared with healthy smokers. b) Serum pro-hepcidin levels from healthy controls $(n=24)$, healthy smokers $(n=22)$ and COPD patients (smokers, $n=19$; ex-smokers, $n=28)$. c) Serum pro-hepcidin levels from healthy controls $(n=24)$, healthy smokers $(n=22)$ and COPD patients stratified by Global Initiative for Chronic Obstructive Lung Disease (GOLD) stage (stage 1, $\mathrm{n}=9$; stage 2, $n=31$; and stage $3, n=6$ ). d) Serum interleukin (IL)-6 levels from healthy controls ( $n=24)$, healthy smokers ( $\mathrm{n}=22$ ) and COPD patients (smokers, $\mathrm{n}=19$; ex-smokers, $\mathrm{n}=28$ ). $\mathrm{b}-\mathrm{d}$ ) ${ }^{* * *}$ : $\mathrm{p}<0.001 ; * *$ : $\mathrm{p}<0.01$; and *: $p<0.05$ when compared with healthy controls. Data were analysed using the Kruskal-Wallis test with Dunn's multiple comparisons post-test.

lung injury or disease [3]. In this regard, the data presented in figure 1a and in particular the obvious and significant discrepancy between redox active iron content in healthy smokers versus COPD smokers, would seem to suggest a failure to excrete iron in the COPD population. Retention of iron within the airways and lungs may well contribute to manifestations of oxidative stress and damage, microbial virulence and altered cellular fate, all of which are of relevance to COPD.

The daily iron requirements of the body far outweigh the ability of the gut to absorb iron from dietary intake, thus most of this limited and vital resource is reprocessed within the body to compensate for this deficit. In global terms iron loss from the body is limited to bleeding and loss of cells from the mucosa including the gut and from sloughing of skin cells. Thus specific body iron excretion mechanisms are not generally well established. However, there is a precedent as performance athletes excrete redox active iron in sweat during exercise [6], which may represent a protective strategy to limit the effects of iron catalysed oxidant production in these circumstances. The airways and lungs are exposed to iron containing components from the atmosphere on a constant basis and are therefore inherently vulnerable to adverse consequences related to iron accumulation. A mechanism whereby iron and iron-containing particulates 
could be processed by cells in order to detoxify and solubilise this transition metal as free ions to be excreted in breath therefore seems plausible and desirable. Macrophages, inflammatory cell types commonly found in lungs and airways, are known cellular centres for iron turnover and control processing mechanisms for iron uptake in various forms. Indeed, alveolar macrophages are known to sequester iron in patients with COPD [7]. Importantly, macrophages also express the cellular iron export protein ferroportin $[8,9]$, as do airway epithelial cells on the apical surface [10], indicating the potential for iron excretion by these cells in this setting. Ferroportin expression is chiefly controlled by the small peptide hormone hepcidin, which when bound to ferroportin causes endocytosis thereby preventing cellular iron export. Hepcidin exists as a preprohormone, a prohormone and the mature 25 amino acid peptide. In the hepatocyte, hepcidin maturation occurs mainly by the prohormone convertase furin $[8,9]$. Pro-hepcidin is biologically inactive $[8,9]$ and its role in serum remains elusive. Hepcidin expression is controlled by both iron availability via the BMP/Smad-dependant signalling pathways and inflammatory stimuli, most notably IL-6 which signals via the JAK/STAT pathway $[8,9]$.

A previous study reported that plasma pro-hepcidin was negatively associated with severity of COPD [11]. Our own findings are at variance with this report as our data clearly demonstrates significantly elevated levels of pro-hepcidin in both smoker and ex-smoker COPD populations when compared with normal healthy controls (figure 1b), with a trend for pro-hepcidin levels to increase with disease severity (figure 1c). In addition, increased serum hepcidin during exacerbation characterised a frequent exacerbation phenotype in COPD patients [12]. Furthermore, within the frequent exacerbation group hepcidin was significantly elevated to a greater extent in patients with a positive compared to negative sputum bacteriology.

Elevated hepcidin would limit ferroportin expression and activity and cellular iron release, a scenario that, if operational in lungs and airways, would favour cellular iron retention over excretion with uncertain consequences. Importantly, another recent study described the prevalence of iron deficiency in COPD cohorts that is driven by inflammation [13]. Our own studies have demonstrated significantly elevated levels of IL-6 in patients with COPD and a current smoking habit (figure 1d). Given the role of IL-6 in the control of iron metabolism and turnover it is not unreasonable to suggest a link between IL- 6 release and disrupted iron homeostasis driven by increased hepcidin levels in these circumstances. Indeed associations between serum hepcidin and IL-6 have been described in both exacerbations and stable COPD [14].

Previous studies have determined total iron content in EBC obtained from patient populations including COPD and asthma [15], which indicated lower total iron return compared with controls; the study presented here is distinct from these as measurements were limited to a specific redox active iron pool. Although observational in nature our data indicates discrepancies in iron handling and excretion in patients with COPD compared with normal healthy and healthy smoker controls. Given the potential for iron to contribute to damage, dysfunction and disease when homeostasis is lost we would suggest that a more in depth analysis of iron handling in COPD both systemically and regionally is warranted.

0

@ERSpublications

Decreased redox active iron in exhaled breath condensate from COPD smokers may indicate an excretion failure http://ow.ly/VA4vM

Sharon Mumby ${ }^{1}$, Junpei Saito ${ }^{1}$, Ian M. Adcock ${ }^{1}$, Kian Fan Chung ${ }^{1}$ and Gregory J. Quinlan ${ }^{2}$

${ }^{1}$ Airway Disease Section, National Heart and Lung Institute, Imperial College London, London, UK. ${ }^{2}$ Vascular Biology Group, National Heart and Lung Institute, Imperial College London, London, UK.

Correspondence: Gregory J. Quinlan, Vascular Biology Group, National Heart and Lung Institute, Imperial College London, Dovehouse Street, London, UK. E-mail: g.quinlan@imperial.ac.uk

Received: Oct 152015 | Accepted after revision: Nov 242015 | First published online: Dec 232015

Support statement: This research was supported by the NIHR Respiratory Disease Biomedical Research Unit at the Royal Brompton and Harefield NHS Foundation Trust and Imperial College London, and by the Royal Brompton Hospital Charitable Fund. Funding information for this article has been deposited with FundRef.

Conflict of interest: Disclosures can be found alongside the online version of this article at erj.ersjournals.com

\section{References}

1 Silverberg DS, Mor R, Weu MT, et al. Anemia and iron deficiency in COPD patients: prevalence and the effects of correction of the anemia with erythropoiesis stimulating agents and intravenous iron. BMC Pulm Med 2014; 14: 24.

2 Chappell SL, Daly L, Lotya J, et al. The role of IREB2 and transforming growth factor beta-1 genetic variants in COPD: a replication case-control study. BMC Med Genet 2011; 12: 24.

3 Mumby S, Chung KF, McCreanor JE, et al. Pro-oxidant iron in exhaled breath condensate: a potential excretory mechanism. Respir Med 2011; 105: 1290-1295.

4 Gutteridge JM, Mumby S, Quinlan GJ, et al. Pro-oxidant iron is present in human pulmonary epithelial lining fluid: implications for oxidative stress in the lung. Biochem Biophys Res Commun 1996; 220: 1024-1027. 
5 Ghio AJ, Hilborn ED, Stonehuerner JG, et al. Particulate matter in cigarette smoke alters iron homeostasis to produce a biological effect. Am J Respir Crit Care Med 2008; 178: 1130-1138.

6 Gutteridge JM, Rowley DA, Halliwell B, et al. Copper and iron complexes catalytic for oxygen radical reactions in sweat from human athletes. Clin Chim Acta 1985; 145: 267-273.

7 Philippot Q, Deslée G, Adair-Kirk TL, et al. Increased iron sequestration in alveolar macrophages in chronic obstructive pulmonary disease. PLoS One 2014; 9: e96285.

8 Miseta A, Nagy J, Nagy T, et al. Hepcidin and its potential clinical utility. Cell Biol Int 2015; 39: 1191-1202.

9 Ruchala P, Nemeth E. The pathophysiology and pharmacology of hepcidin. Trends Pharmacol Sci 2014; 35: $155-161$.

10 Yang F, Haile DJ, Wang X, et al. Apical location of ferroportin 1 in airway epithelia and its role in iron detoxification in the lung. Am J Physiol Lung Cell Mol Physiol 2005; 289: L14-L23.

11 Duru S, Bilgin E, Ardic S. Hepcidin: a useful marker in chronic obstructive pulmonary disease. Ann Thorac Med 2012; 7: 31-35.

12 Mackay AJ, Loza M, Branigan PJ, et al. Serum amyloid A and hepcidin elevations characterize bacterial-associated exacerbations in frequently exacerbating COPD patients. Am J Respir Crit Care Med 2015; 191: A6363.

13 Nickol AH, Frise MC, Cheng HY, et al. A cross-sectional study of the prevalence and associations of iron deficiency in a cohort of patients with chronic obstructive pulmonary disease. BMJ Open 2015; 5: e007911.

14 Tandara L, Grubisic TZ, Ivan G, et al. Systemic inflammation up-regulates serum hepcidin in exacerbations and stabile chronic obstructive pulmonary disease. Clin Biochem 2015; 48: 1252-1257.

15 Mutti A, Corradi M, Goldoni M, et al. Exhaled metallic elements and serum pneumoproteins in asymptomatic smokers and patients with COPD or asthma. Chest 2006; 129: 1288-1297.

\section{Proposed new definition of exercise pulmonary hypertension decreases false-positive cases}

To the Editor:

The exercise part of the haemodynamic definition of pulmonary hypertension $(\mathrm{PH})$ was used between the first (1973) [1] and fourth (2008) [2] world conferences on $\mathrm{PH}$ for patients with a mean pulmonary arterial pressure $(\mathrm{mPAP})>30 \mathrm{mmHg}$ during exercise. However, at the World Symposium on Pulmonary Hypertension in Dana Point in 2008, this part of the PH definition was abandoned, mainly due to the fact that pulmonary arterial pressure (PAP) values during exercise are strongly dependent on age and exercise level [3], and a definition based on a single parameter such as PAP may not be reliable. The analysis of historical studies $[3,4]$ and the generation of novel data [5-8] have led to a better understanding of exercise haemodynamics. Herve et al. [7] suggest a new definition of exercise-PH that includes not only PAP, but also total pulmonary resistance (TPR), calculated as $\mathrm{mPAP} /$ cardiac output, representing the steepness of PAP increase during exercise. According to this definition, exercise-PH may be diagnosed if during maximal exercise $\mathrm{mPAP}>30 \mathrm{mmHg}$ and TPR $>3$ Wood units (WU). This may reduce the number of false positive exercise- $\mathrm{PH}$ diagnoses in healthy subjects who develop high mPAP values at excessive exercise levels.

We applied the old and the suggested new definition of exercise-PH to a historical cohort of normal subjects whose individual invasively assessed haemodynamic data were published in the scientific literature $[3,4]$. In addition, we asked whether the assessment of pulmonary arterial wedge pressure (PAWP) and pulmonary vascular resistance (PVR) during exercise in addition to TPR might provide additional information about subjects with elevated PAP values during exercise.

Our databank contains all invasively assessed haemodynamic data at rest and during exercise of 1187 subjects, which were published in 47 historical studies. We assumed that all subjects were healthy in terms of haemodynamics. Exercise was performed on a cycle ergometer in the supine position in the majority of cases. Only subjects for whom at least mPAP and cardiac output and systemic blood pressure were available at rest and during exercise were included. Subjects with PAWP values at rest and exercise, where PVR could be calculated, were analysed separately. We did not correct or exclude implausible values, which were detected in a few cases. To describe the correlation between MPAP and PAWP as well as PVR and TPR, Spearman rank correlation was used, and $\mathrm{p}<0.05$ was considered statistically significant. 